\title{
Assessing and Adjusting Bias Due to Mixed-Mode in Aspect of Daily Life Survey
}

\author{
Claudia De Vitiis ${ }^{1}$, Alessio Guandalini ${ }^{1}$, Francesca Inglese ${ }^{1}$, and Marco D. Terribili ${ }^{1}$
}

\begin{abstract}
The mixed-mode (MM) designs are adopted by NSIs both to contrast declining response and coverage rates and to reduce the cost of the surveys. However, MM introduces several issues that must be addressed both at the design phase, by defining the best collection instruments to contain the measurement error, and at the estimation phase, by assessing and adjusting the mode effect. In the MM surveys, the mode effect refers to the introduction of bias effects on the estimate of the parameters of interest due to the difference in the selection and measurement errors specific to each mode. The switching of a survey from single to mixedmode is a delicate operation: the accuracy of the estimates must be ensured in order to preserve their consistency and comparability over time. This work focuses on the methods chosen for the evaluation of the mode effect in the Italian National Institute of Statistics (ISTAT) mixed-mode survey "Aspects of Daily Life - 2017", in the experimental context for which an independent control single-mode (SM) PAPI sample was planned to assess the introduction of the sequential web/PAPI survey. The presented methods aim to analyze the causes that can determine significant differences in the estimates obtained with the SM and MM surveys.
\end{abstract}

Key words: Mixed-mode; mode effect; $R$-indicator; instrumental variable approach; propensity score.

\section{Introduction}

The mixed-mode (MM), that is, the use of different collection techniques in the same survey, is a relatively new approach that ISTAT, as well as other NSIs, is adopting especially for social surveys. Its use is spreading both to contrast declining response rates and to reduce the total cost of the surveys (De Leeuw 2005). The use of different data collection modes, in fact, helps in contacting different types of respondents in the most suitable way for each of them, allowing a gain both in population coverage and response rate. However, it introduces a bias, named mode effect, that must be addressed at different levels: in the design phase by defining the best collection instruments to contain the measurement error; in the estimation phase by assessing and treating the bias effects due to the introduction of the mixed-mode data collection, in order to ensure the accuracy of the estimates. The switching of a survey from single to mixed-mode must be planned by aiming to make the MM estimates consistent and comparable with the analogue ones obtained in the previous SM survey editions, to ensure that any changes in the time series are exclusively due to real changes of the observed phenomenon.

\footnotetext{
${ }^{1}$ Italian National Institute of Statistics (ISTAT), Via Cesare Balbo 16, Rome, 00184, Italy. Emails: devitiis@ istat.it, alessio.guandalini@istat.it, fringles@istat.it and terribili@istat.it
} 
Mixed-mode can simultaneously generate nonresponse error (selection effect) and measurement error (measurement effect). Selection effect occurs when different types of respondents choose different modes to answer. This is not actually a problem, but rather a "desirable" consequence of MM surveys. Measurement effect, on the other hand, refers to the influence of a survey mode on the answers that respondents give, such that one person would give different answers in different modes and is caused by differences in the measurement errors specific to each mode. These errors can be due to differences in interviewer effect and social desirability, primacy and recency effects, recall bias, acquiescence, and so on (De Leeuw 2005; Tourangeau and Yan 2007).

The main problem of MM designs is that the selection and measurement effects are confounded, especially when modes are managed in a sequential way. As a consequence, methods for carrying out the inferential process to disentangle the two effects and obtain unbiased estimates of measurement error are needed. The experimental survey designs, as parallel independent samples or re-interview approaches, can facilitate the estimates of the selection and measurement effects (Buelens et al. 2018).

This article focuses on methods for assessing and adjusting mode effect in a survey setting where an independent single-mode survey is carried out together with a MM survey. The methods chosen are applied to the experimental situation of ISTAT "Multipurpose Survey on Households - Aspects of Daily Life (ADL) - 2017” ISTAT (2019). In the 2017 edition, the mixed-mode approach was used for the first time, as a web mode was added to the traditional PAPI mode in a sequential design. A parallel single-mode (SM) PAPI design was planned to allow for an assessment of mode effect on two independent samples collected with different modes. The goal of the analysis presented is the evaluation of the impact on the final estimates of switching from single to mixed-mode. The field of comparison is a survey that produces several indicators for satisfying both national and European requirements. For this purpose, different components of the total non-sampling error are compared in the two samples, as selection effects due to total nonresponse and measurement errors. The total nonresponse is evaluated for both SM and MM samples through the indicators of the representativeness of response (Schouten et al. 2009; Bethlehem et al. 2011; Schouten et al. 2011) and the response rates, using auxiliary variables.

Selection and measurement effects of some survey variables are estimated on the MM respondents set, considering two different approaches: the instrumental variable approach proposed by Vannieuwenhuyze et al. (2010) in the experimental context where a benchmark single-mode survey is available and the propensity score method, generally used in the observational study (Rosenbaum and Rubin 1983; Vandenplas et al. 2016). Finally, a comparison between estimates obtained using different methods for adjusting selection effect is made on the mixed-mode sample (web-PAPI).

The final aim of this article is to point out methods and analyses useful to ensure the accuracy of a complex inferential process for a MM survey, on the basis of evidences on a real survey.

The article is organized as follows: in Section 2 the survey context is outlined, Section 3 describes the outline of the analyses carried out, Section 4 reports methods and results of the comparison between the single and the MM surveys, Section 5 illustrates methods applied to disentangle selection and measurement effects and results, while Section 6 focuses on the adjustment of mode effects. Lastly, Section 7 outlines some concluding remarks. 


\section{Survey and Experimental Context}

The ADL is carried out each year on a selected sample of about 24,000 households. The sample is spread across nearly 850 Italian municipalities using a two-stage sampling design. The sample of households is selected from the centralized municipal register. In the 2017 edition of the ADL survey, a mixed-mode design was introduced for the first time: a web mode has been added to the traditionally used PAPI mode in a sequential design. In order to analysz the impact of the MM on the estimates of the parameters of interest, a survey design was made in which the sample of each municipality was randomly divided to two sub-samples. In the first sub-sample, of larger size (19,262 households), the mixed web/PAPI mode has been administered sequentially (mixed-mode, MM design), while in the second one $(8,014$ households), only the PAPI interview has been proposed (single-mode SM, control sample).

The two surveys started simultaneously with an invitation letter sent by ISTAT, about one month before, to invite the households to participate in the survey. In the PAPI singlemode (SM) survey, filling two questionnaires in paper form is required: the basic survey questionnaire administered face-to-face by a municipal interviewer to all individuals in the household and a self-reported questionnaire delivered by the municipal interviewer to each individual of the household. In the sequential MM survey, ISTAT sent an invitation letter to all sample households inviting them to fill the questionnaires in electronic form via the web, using the credentials given by ISTAT in the presentation letter. If the household did not complete the questionnaire on the web, at the end of the period foreseen for online filling (about two months with two reminder letters), a municipal interviewer personally address the same questionnaires to all the individuals.

\section{Outline of the Analyses of the Mode Effect}

\subsection{The Analyses}

The analyses carried out on the ADL survey aimed firstly to evaluate the impact of the introduction of MM design on the estimate of the parameters of interest with respect to the single-mode design and, subsequently, to analyze in-depth the reasons that determine significant differences in the estimates obtained with the two designs. For this purpose, the study was developed on several levels of analysis, corresponding to different operational steps. The first level is based on the comparison between the two samples (SM and MM); the second level addresses the evaluation of the components of the mode effect (selection and measurement) of the MM design; and the third level consists of some experiments to adjust for the selection effect in the MM response set.

The analyses were developed thanks to the acquisition of mode insensitive auxiliary variables (socio-demographic) that has been obtained through the linkage of the units of SM and MM samples with administrative data performed through the individual code. The administrative database comes from the Archimede Project, (Integrated archive of economic and demographic microdata, Garofalo 2014; Ballabio et al. 2018) built for expanding ISTAT information provided by administrative archives to produce longitudinal paths and cross-sectional collections of microdata to be made available to different users. The auxiliary variables acquired on the individuals selected in the samples are: education level, occupation type, tax income, citizenship, geographical area and municipal type. 
For some analyses, these structural variables were redefined at the household level, as the household as a whole is involved in the response process and the "choice" of the mode. The household auxiliary variables, defined by aggregating the individual ones, are: higher education level (below/equal/above high school diploma); income class (five quintiles - (1) $<$ EUR 10,508, EUR 10,508-20,281, EUR 20,281-29,778, EUR 29,778-46,079, and EUR >46,079); (2) citizenship (Italian/Foreign household); (3) household type (oneindividual under 35, one-individual 35-64, one-individual over 64, two-individuals at least one under 35, two-individuals all over 34, more than two individuals at least one under 25, more than two individuals all of them over 24); (4) municipal type (Metropolitan cities, metropolitan area, other municipalities with $<2,000,2,000-10,000,10,000-50,000$, $>50,000$ inhabitants); (5) different geographical areas, such as Italian regions (NUTS 2) and macro-areas (NUTS 1: North West, North East, Center, South, Islands).

In the first step of the analysis, tests were performed on the differences in the estimates calculated on SM and MM samples, for a set of relevant survey variables, to highlight the variables suspected of being significantly susceptible to mode effect. Subsequent analyses, based on response rates and some indicators of the representativeness of response, were conducted to study the total nonresponse effect in the two samples, considering a set of the household structural variables. The total nonresponse can introduce a bias effect in the survey estimates and compromise their precision level. While the precision of the estimates is directly related to the response rate, the bias does not only depend on the response rate but also on the difference between respondents and non-respondents. To assess the overall quality of the surveys (especially in terms of the magnitude of the bias), two types of indicators of representative response were used, the global R-indicator and the partial unconditional R-indicator (Schouten et al. 2009; Bethlehem et al. 2011; Schouten et al. 2011). Dissimilarities in both response rates and indicators of representative response could explain, at least partly, differences in the estimates of the survey produced with the SM and MM samples, due to the different composition of SM and MM respondents (selection effects) with respect to a given set of auxiliary variables.

In the second step, the assessment of the mode effect in the MM survey was carried out for some target variables following different methods that make the respondent units to the web and PAPI modes comparable: the propensity score (Rosenbaum and Rubin 1983) is applied using only the MM respondents and a PAPI mode as the reference; the instrumental variable approach proposed by Vannieuwenhuyze (2010) is adopted using the PAPI mode of the SM respondents as a benchmark. The MM estimates can be affected either by the different composition of the web and PAPI respondents or by differences in measurement errors generated from each mode (Hox et al. 2015).

In the third step, some experiments to adjust for MM selection effect were made. In particular, the calibration on fixed proportions of the web and PAPI respondents were applied in order to stabilize the total measurement error over time (Buelens and Van den Brakel 2014). This method was compared with other weighting methods, as propensity score and calibration on demographic known totals (individual structural variables).

In the following scheme, the steps and the methods considered in the study are listed. The structure of "Scheme 1" could be used for (at least) two purposes: (1) to present results from a case study (the ADL-experiment in this case); (2) to describe how different methods can be applied when analyzing a mixed-mode experiment. 
Scheme 1. Operational steps of the analysis

\begin{tabular}{|c|c|c|c|}
\hline & Method & Objective & Assumptions/conditions \\
\hline \multirow[t]{4}{*}{$\begin{array}{l}\text { First } \\
\text { step }\end{array}$} & $\begin{array}{l}\text { 1) Tests on the differences in the } \\
\text { estimates calculated on the } \\
\text { two samples for a set of } \\
\text { relevant survey } \\
\text { variables }\end{array}$ & $\begin{array}{l}\text { Highlighting the } \\
\text { variables for which a } \\
\text { suspect of mode effect } \\
\text { was significant }\end{array}$ & $\begin{array}{l}\text { Independence between } \\
\text { the two samples }\end{array}$ \\
\hline & $\begin{array}{l}\text { 2) Tests on the response rates in } \\
\text { the SM and MM sample. }\end{array}$ & $\begin{array}{l}\text { Analysis of the response } \\
\text { processes and evaluation } \\
\text { of the bias }\end{array}$ & $\begin{array}{l}\text { Independence between } \\
\text { the two samples; MAR } \\
\text { assumption for the }\end{array}$ \\
\hline & 3) Response rates and tests & $\begin{array}{l}\text { caused by the total non- } \\
\text { response }\end{array}$ & response models \\
\hline & $\begin{array}{l}\text { 4) Indicators of } \\
\text { representativeness }\end{array}$ & & \\
\hline \multirow[t]{2}{*}{$\begin{array}{l}\text { Second } \\
\text { step }\end{array}$} & $\begin{array}{l}\text { 5) Instrumental variable } \\
\text { approach }\end{array}$ & $\begin{array}{l}\text { Disentangling measure- } \\
\text { ment and selection } \\
\text { effects }\end{array}$ & $\begin{array}{l}\text { Representativity } \\
\text { assumption }\end{array}$ \\
\hline & 6) Propensity score & & $\begin{array}{l}\text { MAR assumption for } \\
\text { the response models; } \\
\text { Balancing assumption }\end{array}$ \\
\hline \multirow[t]{2}{*}{$\begin{array}{l}\text { Third } \\
\text { step }\end{array}$} & $\begin{array}{l}\text { 7) Weighting methods as pro- } \\
\text { pensity score, } \\
\text { calibration }\end{array}$ & To adjust selection effect & $\begin{array}{l}\text { Ignorability of selection } \\
\text { mechanism; } \\
\text { Measurement error } \\
\text { negligible }\end{array}$ \\
\hline & 8) Mode calibration & $\begin{array}{l}\text { To stabilize the total } \\
\text { measurement error over } \\
\text { time }\end{array}$ & $\begin{array}{l}\text { Invariance over time of } \\
\text { measurement error }\end{array}$ \\
\hline
\end{tabular}

\subsection{Analysis Validation: Assessing the Significance of the Estimated Parameters}

To properly assess the estimated parameters and effects, their significance must be studied in view of sampling variance. Due to the complexity of the estimated parameters and the complex sampling design adopted in ADL, bootstrapping (Efron 1979; Efron and Tibshirani 1986; Wolter 2007) would seem the most suitable solution. However, despite its widespread use for variance estimation in statistics, there is some controversy when using a method that implies some modelization.

Drawing bootstrap samples entails incorporating additional potential sources of variability when using methods that rely on models estimated on the original sample. In fact, in each bootstrap sample, the model must be estimated, and therefore, variability due to parameter estimation of the model is added. The device suggested by Austin and Small (2014) can be adapted to this context (for instance, when using $R$-indicators and propensity score). They suggest estimating the model for the whole sample and then selecting bootstrap samples and applying the methods just considering the parameters estimated from the original sample, that is, as the model is given. An alternative method is to use pseudo-replication (Rust and Rao 1996). Each replication is defined by a random weight assigned, according to the sample design, to each unit of the original sample. Also in this case, the model must be estimated just one time and this enables the estimation of the variance without involving additional variance due to the estimation of the model. 
The two replication methods provide very similar results. However, for the present purpose the pseudo-replication method has been used and 500 pseudo-replications of the ADL sample, according to the sample design, have been carried out with the aim of properly assessing the estimated parameters and effects.

\section{First Step: The Comparison Between the Single and the Mixed-Mode Survey}

\subsection{Test of Differences Between Estimates}

Tests on the differences in the estimates between the SM and MM surveys were performed, through chi-square test, to determine whether there are significant differences between the distributions of the answer with respect to the data collection mode and t-test to determine whether the difference between proportions of individuals for each item is significant with respect to the data collection mode (Martin and Lynn 2011). The hypothesis tests concerned numerous survey indicators. Among these variables, the difference for the distributions of the following variables were found to be significant: life satisfaction; reading books in the last year; use of PC; use of internet; trust in others; frequency of seeing friends. Figure 1 shows the detailed distributions for three variables for which the two independent response sets produced estimates showing the most relevant differences between categories (NR is the nonresponse category).

\subsection{Response Rates}

The response rates in the SM and MM surveys are calculated on the list of the households sampled, and deleting non-eligible units and "unresolved" units (Eurostat 2014). In the response rate analysis, some household structural variables were used.

To test whether the differences between the response rates in the two independent samples (SM, MM) are statistically significant, a z-test was used, with a confidence level $1-\alpha=0.99$. In Table 1 the response rates for the two surveys and a $\mathrm{Zc}$ statistic are reported by macro-areas.

The SM and MM surveys achieved different response rates. The response rate was generally slightly higher for the MM survey (74\% versus $71 \%$ of the SM), while for the web mode the response rate varied greatly between Italian macro-areas (ranging from $36 \%$ in the North East to $17 \%$ in the South and Islands).

The response rate results were significantly different in the North-West and the NorthEast. This type of analysis, extended to other structural variables, revealed that the response rates in the two surveys are significantly different when the household presents some characteristics, such as mixed nationality, lower-income class, is composed of just one individual between 35 and 64 years old or of more than two individuals, at least one of them under 26 years old.

Further analyses were conducted to assess whether the response rate distributions are independent of structural variables. The hypothesis of independence between the response and the auxiliary variables was tested through a chi-square test. The results highlighted that these variables influence the response in both surveys, above all the variables that characterize households based on education level, lower income class and mixednationality-households, and where the households live, such as northern metropolitan cities. 


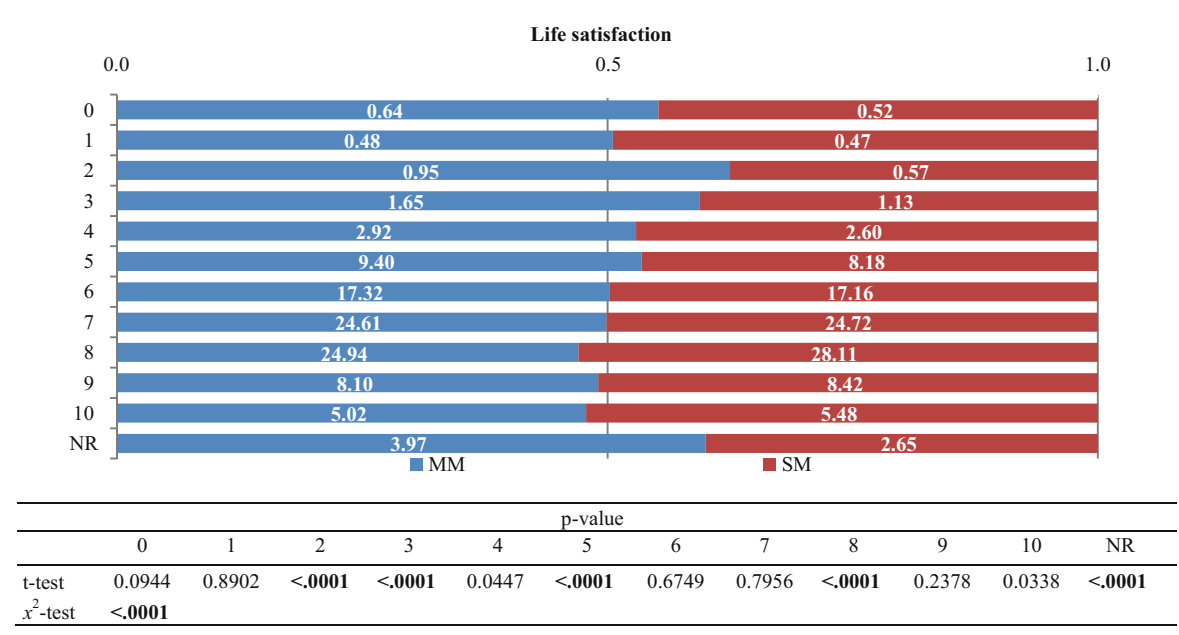

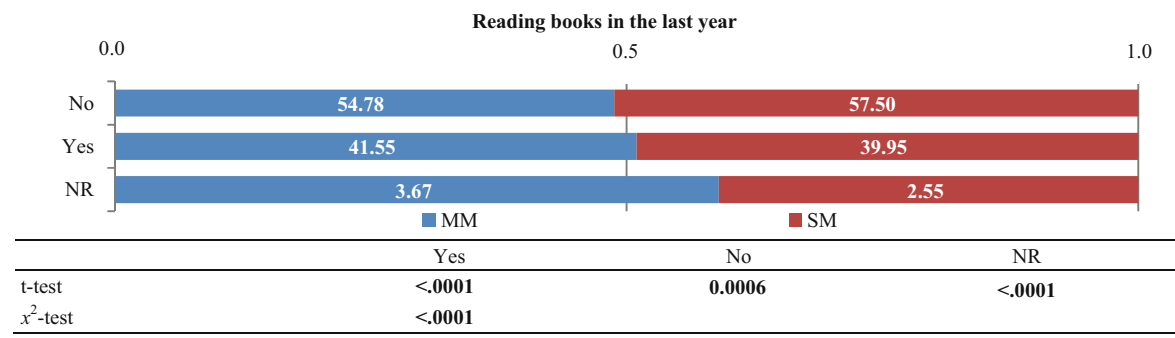

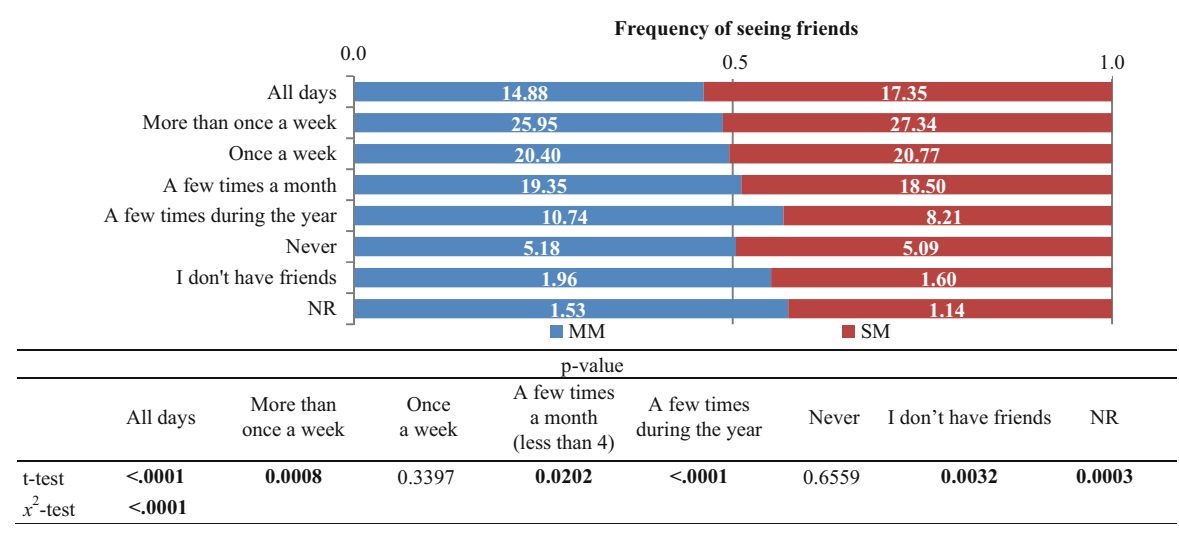

Fig. 1. Distributions of the responses for MM and SM samples for some survey target estimates with p-values of ttest and $\chi^{2}$-test on the distributions. NR is the nonresponse category, significant differences at $95 \%$ are in bold font.

\subsection{Indicators of Representativity}

The indicators of representativity, called $R$-indicators, are based on a measure of the variability of the response propensity, with respect to a given set $X$ of auxiliary variables, known for both respondent and non-respondent units (sample-based auxiliary information). $R$-indicators may measure deviations from representative response with respect to a vector of available $X$. The response is representative with respect to some 
Table 1. Sample size and response rate in the SM and MM surveys by macro-area.

\begin{tabular}{|c|c|c|c|c|c|c|}
\hline \multirow[t]{2}{*}{ Macro area } & \multicolumn{2}{|c|}{ SM } & \multicolumn{2}{|c|}{ Total MM } & \multicolumn{2}{|c|}{ Web in $\mathrm{MM}$} \\
\hline & $\mathrm{n}$ & $\begin{array}{l}\text { Response } \\
\text { rate }\end{array}$ & $\mathrm{n}$ & $\begin{array}{l}\text { Response } \\
\text { rate }\end{array}$ & $Z c$ & $\begin{array}{l}\text { Response } \\
\text { rate }\end{array}$ \\
\hline North West & 1900 & $65.9 \%$ & 4369 & $71.2 \%$ & $-4.193^{*}$ & $32.5 \%$ \\
\hline North East & 1741 & $70.2 \%$ & 4028 & $73.6 \%$ & $-2.657 *$ & $36.0 \%$ \\
\hline Center & 1616 & $68.6 \%$ & 3735 & $70.2 \%$ & -1.169 & $27.8 \%$ \\
\hline South & 1781 & $79.3 \%$ & 5314 & $79.4 \%$ & -0.090 & $17.7 \%$ \\
\hline Islands & 976 & $71.3 \%$ & 1816 & $74.2 \%$ & $-1,649$ & $17.3 \%$ \\
\hline ITALY & 8014 & $71.0 \%$ & 19262 & $74.0 \%$ & $-5,091 *$ & $26.8 \%$ \\
\hline
\end{tabular}

* The difference between SM and Total MM response rates is statistically significant at a confidence level of $99 \%$.

categorical variable $X$ when the average response probabilities over the classes of $X$ are equal (Schouten et al. 2009).

Schouten et al. (2009) propose two indicators of representativity in the population to compare the quality surveys or the quality of the same survey over time that are based on the standard deviation, $S\left(\rho_{X}\right)$, of the response propensities $\rho_{X}$ (the response propensity function for the auxiliary variables $X)$ : the global $R$-indicator, $R\left(\rho_{X}\right)=1-2 S\left(\rho_{X}\right)$, and the unconditional partial $R$-indicator, $P_{u}\left(Z, \rho_{X}\right)=S_{B}\left(\rho_{X} \mid Z\right)$. The first indicator describes how the respondent units reflect the target population according to certain characteristics and measure how much the response set in a survey deviates from the representative response. It takes values on the interval $[0,1]$, with the value 1 indicating the most representative response. The response is not representative if there is much variation in response probabilities, reflected by a large standard deviation.

The unconditional partial $R$-indicator, instead, can be seen as a measure of the impact of the specified variable on deviations from the representative response (Schouten et al. 2011). This indicator measures the distance to representative response for a single auxiliary variable and is based on the between variance of the response propensities given a stratification with categories of $Z$ : it is non-negative and assumes values less than or equal to 0.5. At the subpopulation (category) level it assumes values between -0.5 and 0.5 : a negative value indicates that a subpopulation is under-represented, while a positive value indicates that a subpopulation is over-represented, and the value 0 means that it is represented (Schouten et al. 2011).

The sample-based $R$-indicators are obtained using a response model (logit or probit regression models) and auxiliary variables known for respondents and nonrespondents are used to estimate the response propensity. Response propensity, $\rho_{X}$, is the conditional expectation of $R_{i}$ (response indicator variable which takes the value 1 if unit $i$ responds and the value 0 otherwise) given the vector of values $x_{i}$. of the vector $X$ of auxiliary variables. The estimator of $R$-indicator, $\hat{R}\left(\hat{\rho}_{X}=1-2 \hat{S}\left(\hat{\rho}_{X}\right)\right.$, is obtained using the estimator of the variance of the response propensities, $\hat{S}^{2}\left(\hat{\rho}_{X}\right)=\frac{1}{N-1} \sum_{S} d_{\mathrm{i}}\left(\hat{\rho}_{X}\left(x_{i}\right)-\widehat{\bar{\rho}}_{X}\right)^{2}\left(d_{i}\right.$ is the design weight and $\widehat{\bar{\rho}}_{X}$ is the weighted sample average of the estimated response propensities). The $\hat{R}\left(\hat{\rho}_{X}\right)$ can be used to derive the standardized absolute maximum bias (Bethlehem et al. 2011), $\hat{\mathrm{B}}_{\mathrm{m}}\left(\hat{\rho}_{\mathrm{X}}\right)=\left(1-\hat{R}\left(\hat{\rho}_{X}\right)\right) / 2 \widehat{\bar{\rho}}_{X}$.

The estimator of unconditional partial $R$-indicator, expressed as $\hat{P}_{u}\left(Z, \hat{\rho}_{X}\right)=\hat{S}_{B}\left(\hat{\rho}_{X} \mid Z\right)$, is obtained using the estimator of the between variance, $\hat{S}_{B}^{2}\left(\left(\hat{\rho}_{\mathrm{x}} \mid Z\right)\right)=\sum_{k=1}^{K}$ $\frac{\hat{N}_{k}}{N}\left(\widehat{\overline{\boldsymbol{\rho}}}_{\mathrm{x}, \mathrm{k}}-\widehat{\overline{\boldsymbol{\rho}}}_{\mathrm{x}}\right)^{2}$, given a $K$ strata of variable $Z$ that can be included or not in the model 
for response. At the level of the subpopulation (strata) $Z=k$, the unconditional partial $R$-indicator is $\hat{\mathrm{P}}_{\mathrm{u}}\left(\mathrm{Z}, \mathrm{k}, \hat{\rho}_{\mathrm{x}}\right)=\hat{\mathrm{S}}_{\mathrm{B}}\left(\hat{\rho}_{\mathrm{x}} \mid \mathrm{Z}=\mathrm{k}\right)$, with the estimator of the variance $\hat{\mathrm{S}}_{\mathrm{B}}\left(\hat{\rho}_{\mathrm{x}} \mid \mathrm{Z}=\mathrm{k}\right)=\sqrt{\frac{\hat{N}_{k}}{N}}\left(\widehat{\bar{\rho}}_{\mathrm{x}, \mathrm{k}}-\widehat{\bar{\rho}}_{\mathrm{x}}\right)$.

The estimate of response probabilities through the sample introduces additional precision loss in the $R$-indicator estimates. To evaluate the estimates of the global $R$ indicator and the unconditional partial $R$-indicator, the standard error and the confidence intervals must be calculated.

For the considered survey context, the estimates of the indicators of the representative response are obtained starting from different logistic regression models for response, $\log \left[\rho_{x} /\left(1-\rho_{x}\right)\right]=x^{\prime} \beta$. The models are defined at the national level (Italy) and for three macro-areas (North, Center, South and Islands), considering as dependent variable the response indicators, $R_{S M, i}$ for the SM sample and $R_{M M, i}$ for the MM sample, and as independent variables the vector of the household structural variables. The covariates of the logit models with a significant regression coefficient are: household type, income class, higher educational level and geographical areas (five macro-areas in the model for the national level and the municipal type in the models for the macro-areas).

Tables 2 to 5 present, for both SM and MM samples, the estimates of $\hat{R}\left(\hat{\rho}_{x}\right)$, the values of the standardized absolute maximum bias proposed by Bethlehem et al. (2011), and the related standard error (SE) with the confidence intervals at 95\% (CI 95\%). As already discussed in Subsecon 3.2, standard errors are obtained through the pseudo-replication method to take into account the sample design of the survey and correctly estimate the variance of the $R$-indicators without involving the estimation of the model. Then, confidence intervals are computed using a Gaussian assumption for the sampling variance.

Table 2 shows that, at the national level the MM response set deviates less from the representative response with respect to the SM sample, 0.854 in the first and to 0.812 in the second, confirming a better representativity of the mixed-mode survey. For the North, the values of the $R$-indicator are similar for the two samples, for the other macro-areas they are very different. The response in these cases is more representative when the MM survey is adopted. Although the web response rates are much lower in the South and Islands, it

Table 2. Global R-indicator estimates for the SM and MM samples in the macro-area and at national level.

\begin{tabular}{|c|c|c|c|c|c|c|}
\hline \multirow{2}{*}{$\begin{array}{c}\text { Macro- } \\
\text { area }\end{array}$} & \multicolumn{3}{|c|}{ SM sample } & \multicolumn{3}{|c|}{ MM sample } \\
\hline & $\begin{array}{l}\text { Estimate } \\
\text { (CI 95\%) }\end{array}$ & SE & $\begin{array}{l}\text { Absolute } \\
\text { maximum } \\
\text { bias }\end{array}$ & $\begin{array}{l}\text { Estimate } \\
\text { (CI 95\%) }\end{array}$ & SE & $\begin{array}{c}\text { Absolute } \\
\text { maximum } \\
\text { bias }\end{array}$ \\
\hline North & $\begin{array}{r}0.847 \\
(0.841,0.853)\end{array}$ & 0.00311 & 0.0516 & $\begin{array}{r}0.840 \\
(0.832,0.848)\end{array}$ & 0.00433 & 0.0578 \\
\hline Center & $\begin{array}{r}0.752 \\
(0.744,0.760)\end{array}$ & 0.00428 & 0.0844 & $\begin{array}{r}0.842 \\
(0.832,0.852)\end{array}$ & 0.00491 & 0.0572 \\
\hline $\begin{array}{l}\text { South } \\
\text { and } \\
\text { Islands }\end{array}$ & $\begin{array}{r}0.840 \\
(0.833,0.847)\end{array}$ & 0.00339 & 0.0608 & $\begin{array}{r}0.907 \\
(0.899,0.915)\end{array}$ & 0.00429 & 0.0337 \\
\hline Italy & $\begin{array}{r}0.814 \\
(0.810,0.818)\end{array}$ & 0.00183 & 0.0657 & $\begin{array}{r}0.854 \\
(0.850,0.858)\end{array}$ & 0.00214 & 0.0542 \\
\hline
\end{tabular}


Table 3. Unconditional partial R-indicator estimates for the income class and strata.

\begin{tabular}{|c|c|c|c|c|c|c|}
\hline \multirow{2}{*}{$\begin{array}{l}\text { Variable } \\
\text { Strata }\end{array}$} & \multicolumn{3}{|c|}{ SM sample } & \multicolumn{3}{|c|}{ MM sample } \\
\hline & Estimate & CI $95 \%$ & SE & Estimate & CI $95 \%$ & SE \\
\hline Income class & 0.0032 & $\begin{array}{r}(0.0029 \\
0.0035)\end{array}$ & 0.00016 & 0.0020 & $\begin{array}{r}(0.0017 \\
0.0023)\end{array}$ & 0.00014 \\
\hline$<10508$ & -0.0449 & $\begin{array}{r}(-0.0451 \\
-0.0447)\end{array}$ & 0.00012 & -0.0359 & $\begin{array}{r}(-0.0361 \\
-0.0357)\end{array}$ & 0.00011 \\
\hline $10508-20281$ & -0.0077 & $\begin{array}{r}(-0.0077 \\
-0.0077)\end{array}$ & 0.00001 & -0.0016 & $\begin{array}{r}(-0.0016 \\
-0.0016)\end{array}$ & 0.00000 \\
\hline $20281-29778$ & 0.0142 & $\begin{array}{r}(0.0142 \\
0.0142)\end{array}$ & 0.00002 & 0.0035 & $\begin{array}{r}(0.0035 \\
0.0035)\end{array}$ & 0.00001 \\
\hline $29778-46079$ & 0.0141 & $\begin{array}{r}(0.0141, \\
0.0141)\end{array}$ & 0.00002 & 0.0123 & $\begin{array}{r}(0.0123 \\
0.0123)\end{array}$ & 0.00002 \\
\hline$>46079$ & 0.0261 & $\begin{array}{r}(0.0260 \\
0.0262)\end{array}$ & 0.00004 & 0.0238 & $\begin{array}{r}(0.0237 \\
0.0239)\end{array}$ & 0.00003 \\
\hline
\end{tabular}

Table 4. Unconditional partial R-indicator estimates for the household typology and strata.

\begin{tabular}{|c|c|c|c|c|c|c|}
\hline \multirow{2}{*}{$\begin{array}{l}\text { Variable } \\
\text { Strata }\end{array}$} & \multicolumn{3}{|c|}{ SM sample } & \multicolumn{3}{|c|}{ MM sample } \\
\hline & Estimate & CI $95 \%$ & SE & Estimate & CI $95 \%$ & SE \\
\hline $\begin{array}{l}\text { Household } \\
\text { type }\end{array}$ & 0.0029 & $\begin{array}{c}(0.0027 \\
0.0031)\end{array}$ & 0.00013 & 0.0019 & $\begin{array}{c}(0.0017 \\
0.0021)\end{array}$ & 0.00008 \\
\hline A & -0.0267 & $\begin{array}{r}(-0.0268 \\
-0.0266)\end{array}$ & 0.00007 & -0.0276 & $\begin{array}{r}(-0.0277 \\
-0.0275)\end{array}$ & 0.00006 \\
\hline B & -0.0328 & $\begin{array}{r}(-0.0329 \\
-0.0327)\end{array}$ & 0.00007 & -0.0199 & $\begin{array}{r}(-0.0200 \\
-0.0198)\end{array}$ & 0.00003 \\
\hline $\mathrm{C}$ & -0.0126 & $\begin{array}{r}(-0.0126 \\
-0.0126)\end{array}$ & 0.00002 & -0.0114 & $\begin{array}{r}(-0.0114 \\
-0.0114)\end{array}$ & 0.00001 \\
\hline D & -0.0015 & $\begin{array}{r}(-0.0015 \\
-0.0015)\end{array}$ & 0.00001 & -0.0080 & $\begin{array}{r}(-0.0080 \\
-0.0080)\end{array}$ & 0.00001 \\
\hline E & 0.0219 & $\begin{array}{r}(0.0219 \\
0.0221)\end{array}$ & 0.00003 & 0.0166 & $\begin{array}{r}(0.0166 \\
0.0166)\end{array}$ & 0.00001 \\
\hline $\mathrm{F}$ & 0.0096 & $\begin{array}{r}(0.0096 \\
0.0096)\end{array}$ & 0.00002 & 0.0091 & $\begin{array}{r}(0.0091 \\
0.0091)\end{array}$ & 0.00001 \\
\hline $\mathrm{G}$ & 0.0206 & $\begin{array}{r}(0.0205 \\
0.0207)\end{array}$ & 0.00003 & 0.0155 & $\begin{array}{r}(0.0155 \\
0.0155)\end{array}$ & 0.00001 \\
\hline
\end{tabular}

seems that in the MM survey the response set better reflects the population of interest with respect to certain characteristics used in the models. At the national level, the upper limit of the bias is higher in the SM survey than in the MM survey. Similar results are shown for the Center and the South and Islands.

The unconditional partial $R$-indicators, $\hat{P}_{u}\left(Z, \hat{\rho}_{X}\right)$ and $\hat{\mathrm{P}}_{\mathrm{u}}\left(\mathrm{Z}, \mathrm{k}, \hat{\rho}_{\mathrm{x}}\right)$, for $\mathrm{SM}$ and MM samples are calculated considering the logistic regression model for response defined at the national level (Italy) to evaluate the contribution to the representativeness of the response of the variables "income class", "household type" and "higher educational level".

The unconditional partial $R$-indicator for "income class" assumes a higher value in the SM sample rather than in the MM sample: this means that the variable contributes more to the non-representativeness of the response in the SM sample. At subpopulation (strata) 
Table 5. Unconditional partial R-indicator estimates for the household typology and strata.

\begin{tabular}{|c|c|c|c|c|c|c|}
\hline \multirow{2}{*}{$\begin{array}{l}\text { Variable } \\
\text { Strata }\end{array}$} & \multicolumn{3}{|c|}{ SM sample } & \multicolumn{3}{|c|}{ MM sample } \\
\hline & Estimate & CI 95\% & SE & Estimate & CI 95\% & SE \\
\hline $\begin{array}{l}\text { Higher } \\
\quad \text { educational } \\
\quad \text { level }\end{array}$ & 0.0002 & $\begin{array}{r}(-0.0002 \\
0.0006)\end{array}$ & 0.00020 & 0.0003 & $\begin{array}{r}(-0.0002 \\
0.0008)\end{array}$ & 0.00025 \\
\hline A & 0.0111 & $\begin{array}{r}(0.0111 \\
0.0111)\end{array}$ & 0.00002 & 0.0120 & $\begin{array}{r}(0.0120 \\
0.0120)\end{array}$ & 0.00002 \\
\hline B & 0.0049 & $\begin{array}{r}(0.0049 \\
0.0049)\end{array}$ & 0.00001 & 0.0111 & $\begin{array}{r}(0.0111, \\
0.0111)\end{array}$ & 0.00002 \\
\hline $\mathrm{C}$ & -0.0034 & $\begin{array}{r}(-0.0043 \\
-0.0025)\end{array}$ & 0.00038 & -0.0030 & $\begin{array}{l}-0.0089 \\
-0.0081)\end{array}$ & 0.00023 \\
\hline
\end{tabular}

level, it can be noted that for the first and second quintiles of the distribution (lower incomes) there is an under-representation of the two samples, although it is more marked for the respondents of the SM survey. In addition, there is a greater over-representation of households with higher incomes in the SM survey (Table 3).

Table 4 shows the results of the unconditional partial $R$-indicator for "household type" and the following categories: one-individual $<=34$ (A); one-individual 35-64 (B); one-individual $>=65(\mathrm{C})$; two-individuals at least one $<=35$ (D); two-individuals all $>34$ (E); more than two individuals at least one $<=24(\mathrm{~F})$; more than two individuals all $>24(\mathrm{G})$.

According to the indicator, the variable "household type" contributes more to the nonrepresentativeness of the response in the SM sample (0.00291) than in the MM sample (0.00195). For the subpopulations, it should be noted that for households with oneindividual and two individuals aged less than or equal to 35 years (A and D) there is an under-representation of the two response sets, although it is more accentuated for the SM survey except for the last subpopulation. There is still more over-representation for households with two individuals over 34 (E) and households with more than two individuals (F and $\mathrm{G}$ ) in the SM survey compared to the MM survey (Table 4).

Table 5 refers to the results of the unconditional partial $R$-indicator for the variable "higher educational level" and the categories below high school diploma (A), equal high school diploma (B) and above high school diploma (C). Table 5 shows the contribution to the representative response of the modality "higher educational level", on the total. It is very low in both response sets. However, it is always higher (in absolute value) in the MM survey than in the SM survey. At the subpopulations level, it should be noted that for households with equal high school diploma (B) there is an over-representation which more accentuated in the MM survey.

\section{Second Step: Evaluation of Mode Effect in the MM Sample}

\subsection{Instrumental Variable Approach}

The instrumental variable approach proposed by Vannieuwenhuyze et al. (2010) is useful for the evaluation of both selection and measurement effects in the experimental context with parallel survey designs and a reference mode. The method requires the validity of the comparability and representativity assumptions and the equivalence of the measurement errors between the reference mode and the same mode used in the mixed-mode design. 
The instrumental variable approach has been adopted starting from two comparable response sets (SM and MM), in which the SM survey (only PAPI mode) is used as a benchmark. In order to make the SM and MM response sets comparable, that is, to ensure that they are not affected by selection effects due to different response processes, a calibration procedure is adopted separately. That is, the sampling weights are modified to match the distributions of socio-demographic known totals (age class, sex, educational level) at the regional level. Then, on the response set of the SM and MM surveys with adjusted weights, the probability distributions of the survey variable are estimated introducing the instrumental variable $I$, which identifies the respondents of the SM and the MM surveys $\left(I_{S M}, I_{M M}\right)$, and the variable $M$, that classifies the respondents in the MM survey according to PAPI and web modes (binomial variable that assumes value 1 for PAPI and 0 for web). The instrumental variables $I_{S M}$ and $I_{M M}$ each have a multinomial

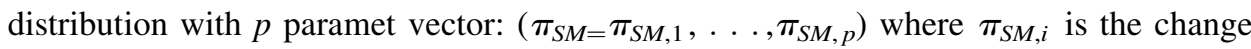
that $I_{S M}=i$ with $0 \leq \pi_{S M, i} \leq 1$ and $\sum \pi_{S M, i}=1$ for $(i=1, \ldots, p) ;\left(\pi_{M M=} \pi_{M M, 1},-\right.$ $\left.\pi_{M M}=\pi_{M M, 1}, \ldots, \pi_{M M, p}\right)$ where $\pi_{M M, i}$ is the change that $I_{M M}=i$ with $0 \leq \pi_{M M, i} \leq 1$ and $\sum \pi_{M M, i}=1$ for $(i=1, \ldots, p)$.

The frequency distributions for $I_{S M}$ and $I_{M M}$, estimated (calibration weights) from both SM and MM response sets are: the distribution of the PAPI responses from SM, $P\left(I_{S M}\right.$; the distribution of the PAPI responses from MM, $P\left(I_{M M} \mid M=1\right)$; the distribution of the web responses from MM, $P\left(I_{M M} \mid M=0\right)$; the distributions of the PAPI and the web responses from all $\mathrm{MM}, P(M=1), P(M=0)$.

From these distributions, it is not possible to estimate the selection and measurement effects. In fact, should be necessary to include the not observed distribution $P\left(I_{S M} \mid M=0\right)$, which represents the counterfactual distribution as if the PAPI respondents in the MM had responded to the web mode (PAPI to web-MM). Starting from the observed distributions, through the total probability formula, $P\left(I_{S M}\right)=P\left(I_{S M} \mid M=0\right) \quad P\left(I_{S M}\right)+P\left(I_{S M} \mid M=1\right) \quad P(M=1)$, Vannieuwenhuyze et al. (2010) derive the distribution, $P\left(I_{S M} \mid M=0\right)=P\left(I_{S M}\right) \frac{1}{P(M=0)}$ $-P\left(I_{S M} \mid M=1\right) \frac{P(M=1)}{P(M=0)}$. From this equation, as $P\left(I_{S M}=i\right)=\pi_{S M, i}$, they obtain for each category of $I_{S M}$, the probabilities $\pi_{S M, i} \mid M=0$ for the respondents who chose the web mode.

The estimate of the selection effect is obtained as the difference between the PAPI-MM and the PAPI to web-MM probability distributions, while the estimate of the measurement effect is obtained as the difference between the web-MM and the PAPI to web-MM probability distributions.

The following tables (Table 6 and Table 7) show the results of the application of the instrumental variable approach for some target variables of the ADL survey, such as "reading books in the last 12 months" and "use of PC in the last year".

Table 6 shows the presence of both selection and measurement effects. The positive selection effect for the No category may be indicative of the fact that the PAPI respondents in the MM sample are more likely to respond No than the web respondents.

Concerning the "Use of PC" (Table 7), for the first category both measurement and selection effects are negative, while with respect to the other categories, they are positive. More individuals respond to other categories when this question is asked in the web mode. The positive selection effect for all categories, except to the first category, indicates that the PAPI respondents in the MM sample are more likely to provide these answers then the web respondents. 
Table 6. Probability distributions of reading books in the last 12 months and estimate of the selection and measurement effects.

\begin{tabular}{lccccrr}
\hline Category & web-MM & PAPI-MM & PAPI-SM & $\begin{array}{c}\text { PAPI to } \\
\text { web-MM }\end{array}$ & $\begin{array}{r}\text { Selection } \\
\text { effect }\end{array}$ & $\begin{array}{c}\text { Measurement } \\
\text { effec }\end{array}$ \\
\hline No & 0.4086 & 0.6291 & 0.5754 & 0.4813 & 0.1478 & -0.0727 \\
Yes & 0.5531 & 0.3348 & 0.3989 & 0.5115 & -0.1767 & 0.0416 \\
Nonresponse & 0.0383 & 0.0361 & 0.0256 & 0.0073 & 0.0288 & 0.0311 \\
\hline
\end{tabular}

Table 7. Probability distributions of Use of PC and estimate of the selection and measurement effects.

\begin{tabular}{|c|c|c|c|c|c|c|}
\hline Category & web-MM & PAPI-MM & PAPI-SM & $\begin{array}{l}\text { PAPI to } \\
\text { web-MM }\end{array}$ & $\begin{array}{l}\text { Selection } \\
\text { effect }\end{array}$ & $\begin{array}{c}\text { Measurement } \\
\text { effect }\end{array}$ \\
\hline $\begin{array}{l}\text { Yes, in the last } \\
3 \text { months }\end{array}$ & 0.6430 & 0.4331 & 0.5279 & 0.6945 & -0.2614 & -0.0515 \\
\hline $\begin{array}{l}\text { Yes, from } 3 \\
\text { months to } 1 \\
\text { year ago }\end{array}$ & 0.0321 & 0.0265 & 0.0260 & 0.0251 & 0.0014 & 0.0070 \\
\hline $\begin{array}{c}\text { Yes, more than } \\
1 \text { year ago }\end{array}$ & 0.0595 & 0.0501 & 0.0484 & 0.0455 & 0.0045 & 0.0140 \\
\hline Never & 0.2293 & 0.4559 & 0.3734 & 0.2285 & 0.2273 & 0.0008 \\
\hline Nonresponse & 0.0360 & 0.0345 & 0.0243 & 0.0063 & 0.0282 & 0.0297 \\
\hline
\end{tabular}

\subsection{Propensity Score Method}

The propensity score (PS) stratification (Rosenbaum and Rubin 1983) can be used to assess the selection and measurement effects. This approach is adopted in observational studies by achieving a balance of covariates between comparison groups. In MM surveys the propensity score can be interpreted as the probability of mode assignment conditional on observed covariates. With adjustments based on PS, the confounding effects of the selection mechanism are mitigated.

The application of this method implied: an estimation of the propensity score model parameters; the definition of subclassification (strata) of respondents based on propensity score; the validation of the balancing assumption, through a chi-square test of the independence between the mode choice and each covariate; for each balanced group, the calculation of weights that equate the weighted proportion of web respondents with the proportion of PAPI respondents in the same stratum.

A logit regression model was used in which the binary response variable is the mode choice web/PAPI is the dependent variable and the structural household variables are the covariates of the model. The covariates of the logit model with a significant regression coefficient are: Italian region, type of municipality, household typology, income class and higher educational level. For eight out of ten of the deciles of the distribution of the predicted probabilities, the independence hypothesis was accepted for all variables.

For each balanced group $k$, a correction factor, or weight, of the selection effect has been calculated as $w_{k}=\frac{n_{k, P A P I} / n_{P A P I}}{n_{k, w e b} / n_{w e b}}$ (Vandenplas et al. 2016), being $n_{k, T}$ the number of respondents to the mode T (web or PAPI) in the group $k$. This corrector allows an overall evaluation of the mode effect in the balanced classes: the selection effect is obtained for each target variable, following Vandenplas et al. (2016), as the difference between the web responses adjusted with $w_{k}$ and the unadjusted web responses, while the measurement effect is obtained as the 
difference between the web responses adjusted with $w_{k}$ and the unadjusted PAPI responses. In Table 9 (see Appendix, Section 8) the majority of the estimated effects are significantly different from zero $(\alpha=0.05)$, based on the bootstrap simulation (see details in Subsection 3.2). What can be underlined from Table 9 is that for many of the items of the selected indicators both the effects are relevant.

For the variable "Reading books in the last 12 months" the results of the estimation for selection and measurement effects obtained through the application of the instrumental approach (Table 6) and the propensity score (Table 9) are similar. The two results show the existence of both a selection and a measurement effect in the same direction. However, for the other variable "Use of PC" (Table 7 and Table 9) the results are not similar. A possible explanation is that this variable is strongly influenced by the propensity of web response: the calibration variables used in the first approach to make the samples comparable could be not sufficiently effective, while the propensity score based on auxiliary variables could be more effective in defining comparable groups of respondent units.

\section{Third Step: Adjusting Mode Effect in the MM Sample}

\subsection{Weighting Methods}

In this section, some methods for adjusting mode effect are applied: the weighting methods, as propensity score and calibration, are used to correct the selection effect.

Generally, the weighting methods assume that the selection effect is ignorable and the measurement error due to the mix of techniques is negligible or at least constant over time, so as not to affect the estimates of variation. The assumption of the invariance over time of measurement error in repeated sequential MM surveys is not very sustainable, because the composition of the respondents by mode can change over the years, leading to variations in the total measurement error.

To avoid the misinterpretation of variations in the composition of respondent samples as variations in the estimates, a calibration procedure that takes into account fixed levels of mode proportions is used. This method is proposed by Buelens and Van den Brakel (2014) and aims to keep the measurement error constant over the survey occasions. The calibration procedure simultaneously performs with respect to both auxiliary variables, that correct the selection effect, and to fixed levels of proportions of response by mode, that stabilize the total measurement error.

Alternative weighting adjustment methods (standard calibration and propensity score subclassification adjustment) are implemented and compared with the previous one.

\subsection{Results}

Table 8 shows the comparison of the estimates of the distribution of "Reading books" that derive from the application of different methods.

These methods are based on calibration procedures that involve distributions of the same socio-demographic totals (age class. sex. educational level) at geographical area level, but that differ for other aspects of the procedure: (1) calibration on only sociodemographics; (2) calibration on socio-demographics and observed fixed levels of mode proportions by six municipal typologies; (3) calibration on socio-demographics and 
Table 8. Estimate of the distribution of "Reading books in the last 12 months" with different methods.

\begin{tabular}{llrrrrr}
\hline Variable & Category & \multicolumn{5}{c}{ Estimate (\%) } \\
\cline { 3 - 7 } & & SM estimate & Meth. 1 & Meth. 2 & Meth. 3 & Meth. 4 \\
\hline Reading & No & 57.81 & 59.92 & 59.00 & 58.66 & 59.92 \\
books (last & Yes & 39.68 & 36.51 & 37.43 & 37.73 & 36.33 \\
12 months) & Nonresponse & 2.49 & 3.58 & 3.56 & 3.61 & 3.75 \\
\hline
\end{tabular}

hypothesized fixed levels (assuming for future occasions an increase of the web response rate in the South and Islands) of mode proportions by six municipal typologies; and (4) calibration on socio-demographics with sampling weights corrected for the web selection effect through correction factors deriving from propensity score (see Subsection 5.2).

What emerges from the table is that the two calibrations including the constraints with respect to the fixed level of mode proportions (methods 2 and 3) determine a difference in the estimate of about $1 \%$.

\section{Concluding Remarks}

The set of the analyses presented can be considered as a possible framework of steps, usable by researchers of NSIs to carry out an assessment of mode effect in similar situations. They try to cover all the different approaches applicable in this specific survey context, even though they do not claim to be exhaustive.

The analyses carried out highlight several issues linked to the introduction of the mixedmode in a social survey. The results show that in MM surveys, the bias due to the total nonresponse is reduced. However, it is still cumbersome to get an overall evaluation of the total measurement error based on different conflicting factors, such as the response process and the mode choice. In fact, it is not easy to under]stand if the different effects are correctly disentangled and estimated.

The analyses conducted on the experimental context of the ADL survey highlight that the MM design helps to better catch the overall population and all the subpopulations that compose it, than the single-mode design. Anyway, the positive impact of MM in terms of obtaining a less selective response does not necessarily improve the accuracy of the estimates. The quality of some of the produced estimates seems affected by a measurement effect.

The results of the analyses conducted on many target variables in the survey, only partially reported in this article, can provide useful advice in planning a further edition of the ADL survey, in order to benefit from the coverage improvement derived from mixing the techniques. In fact, appropriate actions to prevent measurement errors in the survey design phase can make it possible to applly the calibration procedure proposed by Buelens and Van den Brakel (2014) to control for the estimate changes in the time series due to the variation in the composition of web and PAPI respondents (selection effect).

The application of all the methods presented is subject to two important conditions: (1) the availability and the quality of the auxiliary information, which should be mode insensitive and properly explain the selection effect; (2) the validity of the hypotheses underlying the methods, which must be verified by the researcher as far as possible.

In conclusion, the mode effect analyses in a MM survey imply an underlying effort that is hardly compatible with the usual resources and the timing of a statistical process: such 
efforts are challenging and demanding and often there is no time to make them. In general, in order to limit the measurement effect, which is the main drawback of the mixed-mode, in advance as much as possible, accurate planning at the data collection phase is advisable.

\section{Appendix}

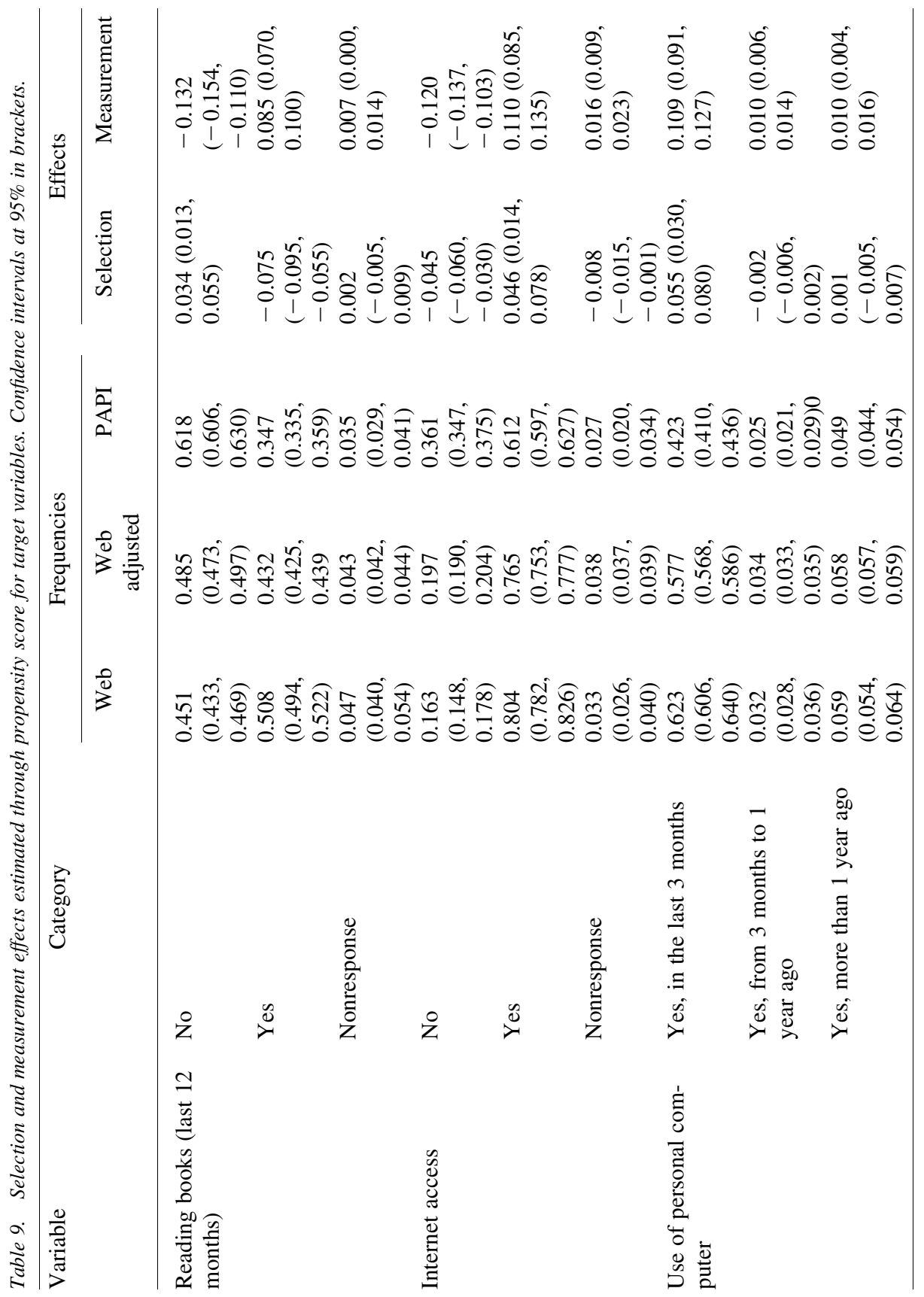




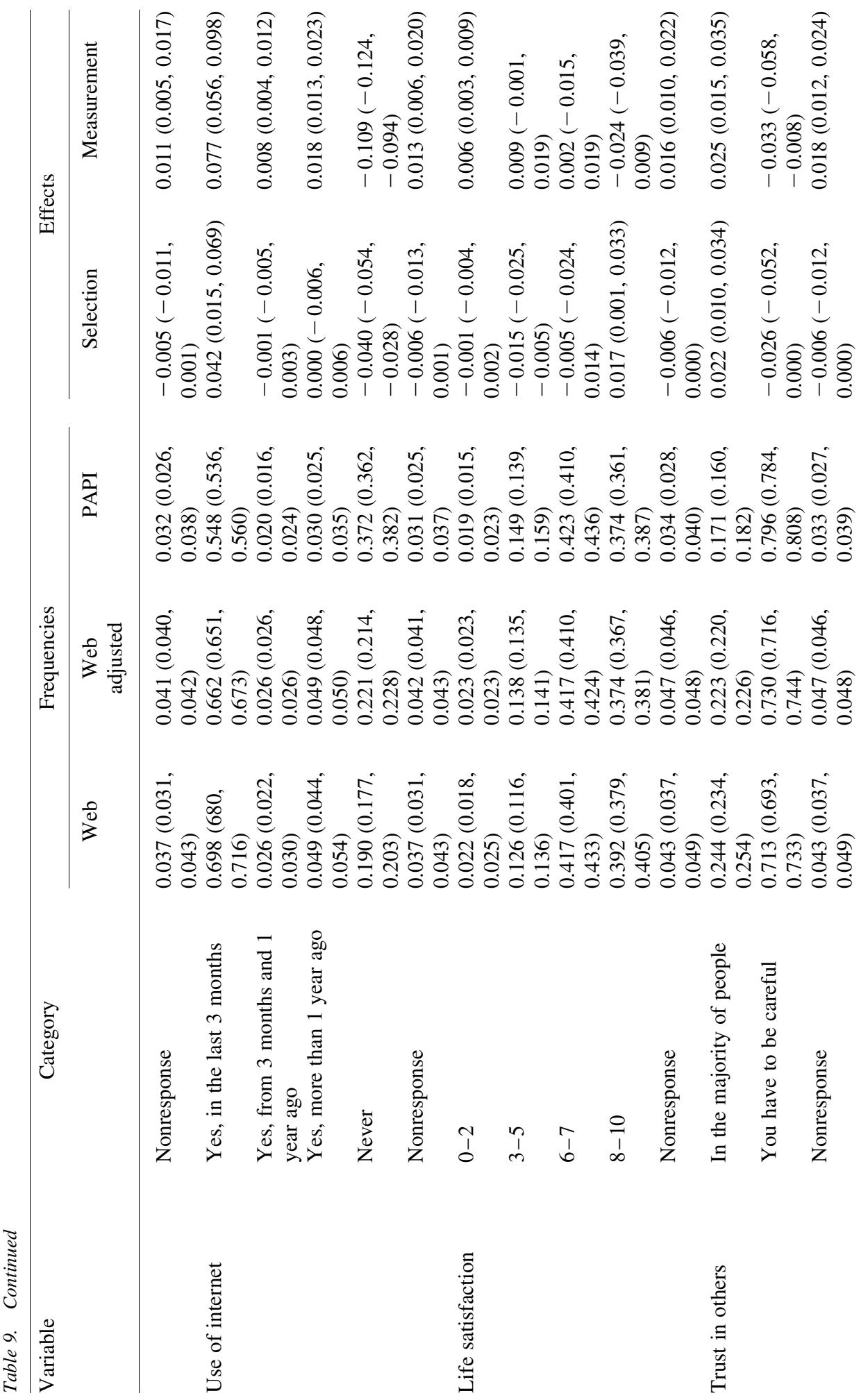




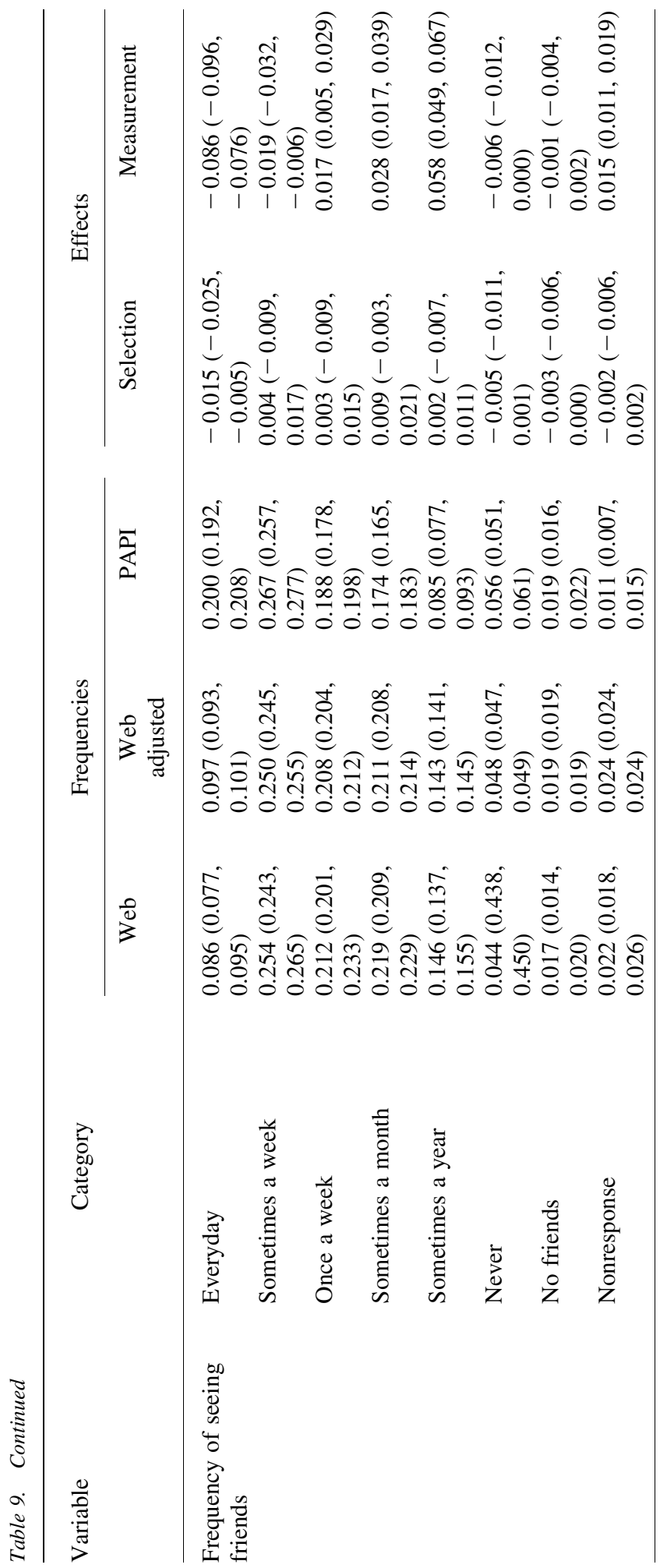




\section{References}

Austin, P.C., and D.S. Small. 2014. "The use of bootstrapping when using propensityscore matching without replacement: a simulation study." Statistics in Medicine 33(24) : 4306-4319. Wiley Online Library. DOI: https://doi.org/10.1002/sim.6276.

Ballabio, S., A. Carra, S. Casacci, D. Ferrazza, F. Verrecchia, A. Vitalini, and L.C. Viviano. 2018. "Local decisions and new guidelines of the Official Statistics." In Proceedings of Q2018 European Conference on Quality in Official Statistics, June 2018, Cracovia, Poland.

Bethlehem, J., F. Cobben, and B. Schouten. 2011. Handbook of nonresponse in household surveys. Wiley Series in Survey Methodology. Hoboken, New Jersey: John Wiley \& Sons, Inc. DOI: https://doi.org/10.1002/9780470891056.

Buelens, B., and J.A. van den Brakel. 2014. "Measurement error calibration in mixedmode Sample Surveys.” Sociological methods and Research 44(3) : 391-426. DOI: https://doi.org/10.1177/0049124114532444.

Buelens, B., J.A. van den Brakel, and B. Schouten. 2018. "Current methodologies to deal with mode effects and mode bias in multi-mode designs." MIMOD Deliverable 1 WP2. Available at: https://www.istat.it/en/research-activity/international-researchactivity/essnet-and-grants (accessed May 2021).

De Leeuw, E.D. 2005. "To mix or not to mix data collection modes in surveys.” Journal of Official Statistics 21(2) : 233-255. Available at: https://www.scb.se/contentassets/ca21efb41fee47d293bbee5bf7be7fb3/to-mix-or-not-to-mix-data-collection-modes-insurveys.pdf (accessed May 2021).

Efron, B. 1979. "Bootstrap methods: Another look at the jackknife." The Annals of Statistics 7(1) : 1-26. DOI: https://doi.org/10.1214/aos/1176344552.

Efron, B., and R. Tibshirani. 1986. "Bootstrap methods for standard errors, confidence intervals, and other measures of statistical accuracy." Statistical Science 1(1) : 54-75. DOI: https://doi.org/10.1214/ss/1177013815.

Eurostat. 2014. ESS Guidelines for the Implementation of the ESS Quality and Performance Indicators (QPI). Luxembourg ESTAT / D4/LA D (2014). Available at: https://ec.europa.eu/eurostat/documents/64157/4373903/02-ESS-Quality-and-performance-Indicators-2014.pdf/5c996003-b770-4a7c-9c2f-bf733e6b1f31 (accessed May 2021).

Garofalo, G. 2014. "Il Progetto ARCHIMEDE obiettivi e risultati sperimentali." Istat Working Paper. Available at: https://www.istat.it/it/files/2014/11/IWP-n.-9-2014.pdf (accessed February 2015).

Hox, J., E.D. de Leeuw, and T. Klausch. 2015. "Mixed Mode Research: Issues in Design and Analysis.” In Total Survey Error in Practice, edited by P.P. Biemer et al.: 511-530. New York: John Wiley \& Sons. DOI: http://dx.doi.org/10.1002/9781119041702.ch23.

ISTAT (2019). “Aspetti metodologici dellindagine. Aspetti della vita quotidiana Periodo di riferimento: anno 2017”. Rome: ISTAT. Available at: https://www.istat.it/microdata/download.php?id=/import/fs/pub/wwwarmida/60/2017/01/Nota.pdf (accessed May 2021).

Martin, P., and P. Lynn. 2011. "The effects of mixed mode survey designs on simple and complex analyses." ISER Working Paper Series 2011-28. Available at: https://ideas. repec.org/p/ese/iserwp/2011-28.html (accessed May 2021). 
Rosenbaum, P.R., and D.B. Rubin. 1983. "The Central Role of the Propensity Score in Observational Studies for Causal Effects.” Biometrika 70: 41-55. DOI: https://doi.org/ 10.1093/biomet/70.1.41.

Rust, K.F., and J.N.K. Rao. 1996. "Variance estimation for complex surveys using replication techniques." In Statistical methods in medical research 5(3) : 283-310. DOI: https://doi.org/10.1177\%2F096228029600500305.

Schouten, B.F., F. Cobben, and J. Bethlehem. 2009. "Indicators for the representativeness of survey response.” In Survey Methodology 35(1) : 101-113. Available at: Indicators for the representativeness of survey response (statcan.gc.ca).

Schouten, B., N. Shlomo, and C. Skinner. 2011. "Indicators for Monitoring and Improving Representativity of Response." Journal of Official Statistics 27: 231-253. Available at: https://www.scb.se/contentassets/ca21efb41fee47d293bbee5bf7be7fb3/indicators-formonitoring-and-improving-representativeness-of-response.pdf (accessed May 2021).

Tourangeau, R., and T. Yan. 2007. "Sensitive questions in surveys." Psychological Bulletin 133(5) : 859-883. DOI: https://doi.org/10.1037/0033-2909.133.5.859.

Vandenplas, C., G. Loosveldt, and J.T.A. Vannieuwenhuyze. 2016. "Assessing the use of mode preference as a covariate for the estimation of measurement effects between modes. A sequential mixed mode experiment." Methods, data, analyses (mda) 10(2) : 119-142. DOI: https://doi.org/10.12758/mda.2016.011.

Vannieuwenhuyze, J.T A., G. Loosveldt, and G. Molenberghs. 2010. "A Method for Evaluating Mode Effects in Mixed-mode Surveys.” Public Opinion Quarterly 74(5) : 1027-1045. DOI: https://doi.org/10.1093/poq/nfq059.

Wolter, K.M. 2007. Introduction to variance estimation. Statistical for Social and Behavioral Science. New York: Springer Science \& Business Media, LLC.

Received June 2019

Revised February 2020

Accepted October 2020 